\title{
3-D analysis of F-actin in stereocilia of cochlear hair cells after loud noise exposure *
}

\author{
Gopal B. Avinash, Alfred L. Nuttall and Yehoash Raphael \\ Kresge Hearing Research Institute. The Unilersity of Michigan, Ann Arbor, Michigan, USA \\ (Received 24 July 1992; Revision received 13 January 1993: Accepted 16 January 199.3)
}

\begin{abstract}
Fluorescence microscopy can be a useful tool in the early detection of pathological changes in the stereocilia of outer hair cells which have undergone acoustic overstimulation. Fluorescent phalloidin, a highly specific F-actin stain, can be used to label F-actin in stereocilia. In this study, phalloidin label is used to determine quantitative changes of F-actin in the stereocilia of guinea pigs exposed to loud noise (117 dB; octave band noise, centered at $1 \mathrm{kHz} ; 4 \mathrm{~h}$ ). Reliably determining three-dimensional (3-D) structural changes in stereocilia is a challenging problem in optical microscopy since stereocilia diameter is close to the optical resolution limit. In order to alleviate the problem, a computational 3-D microscopy technique is used (Avinash et al., 1992). Whole-mounts of the cochlear second and third turns were examined in a Leitz Orthoplan microscope through a Leitz Plan Apo objective lens (100x: 1.32 N.A.; 170/0.17). Images were acquired with a charge-coupled device camera where the focus was shifted in $0.2 \mu \mathrm{m}$ steps using a piezoelectric translator. Images were processed with the appropriate point spread function of the optical system. Analysis of control cochleas indicate that our technique can resolve single stereocilia and distinguish between various intensities of label along each stereocilia. In noise-exposed cochleas, our data show length and intensity changes in the phalloidin label. These results suggest that both depolymerization and polymerization of F-actin can occur in stereocilia of outer hair cells after acoustic overstimulation. Our findings demonstrate the applicability of computational 3-D microscopy to quantitative and qualitative analysis of stereocilia.
\end{abstract}

3-D optical microscopy; Image processing. F-actin; Stereocilia: Cochlear hair cell; Loud noise

\section{Introduction}

Stereocilia in cochlear hair cells (HCs) are typically 2-10 $\mu \mathrm{m}$ long, $0.1-0.3 \mu \mathrm{m}$ wide, and contain actin in a paracrystalline array (Flock and Cheung, 1977). Actin filaments and cross links in stereocilia probably account for their mechanical rigidity. Each actin filament (F-actin) is a polymer composed of polarized monomeric subunits of globular actin (G-actin). Individual filaments are bound together in stereocilia by cross links. Actin filaments may grow by addition of subunits to both ends, but the rate of growth of actin filaments is 5-10 times faster at the positive end than at the negative end (Korn et al., 1987). In nonmuscle cells, polymerized actin exists in equilibrium with the monomeric form under steady state conditions.

Much attention has been given to changes in stereocilia following acoustic trauma, since early signs of damage could be detected in these structures during

Correspondence to: Yehoash Raphael, Kresge Hearing Research Institute, 1301 E. Ann Street, Ann Arbor, MI 48109-0506, USA. Fax: (313) $764-0014$

* Parts of the study were presented at the Fifteenth Midwinter Meeting of the Assocation for the Research in Otolaryngology. (ARO), St. Petersburgh. FL, February 1992. and after acoustic overstimulation. Investigators have reported fused, disarrayed, blebbed, bent, elongated, and missing stereocilia after noise exposure (for review see Saunders et al., 1991). Depolymerization of actin in alligator lizard (Tilney et al., 1982), fractures of rootlets in the cuticular plate in cat (Liberman, 1987), and shortening of the supracuticular rootlet in outer hair cells (OHCs) in cat (Liberman and Dodds, 1987) have been observed.

All of the above results pertaining to acoustic damage to stereocilia were obtained with either scanning electron microscopy or transmission electron microscopy. Preparation of tissue for electron microscopy involves complete dehydration of specimens and, can result in distortion of shape and size of specimens. In addition, electron microscope methods are limited in their ability to provide three-dimensional (3-D) information on localization at the molecular level. In contrast, fluorescence microscopy enables one to localize different molecules in the tissue and does not involve dehydration-related artifacts. In addition, analysis with fluorescence microscopy can be applied on wholemounts of the cochlear spiral, providing a view of the entire population of $\mathrm{HCs}$. Thus, considering that changes in the actin cytoskeleton are among the carly pathologies occurring in $\mathrm{HCs}$ in response to acoustic overstimulation (Raphael and Altschuler, 1991; Saun- 
ders et al., 1985; Tilney et al., 1982), fluorescence microscopy is an attractive method for studying the distribution and organization of actin in HCs.

In fluorescence microscopy, intensity of label at any given point is contaminated by the contributions from the intensity of the label of neighboring points. This problem is especially severe when the specimen is thick and the resolution of the $\mathrm{Z}$-axis is contaminated by out-of-focus signal. We have developed a computational 3-D microscopy technique to overcome the limitation of blur in fluorescence microscopy.

The main goal of this paper is to demonstrate the applicability of our computational technique to assay the effects of acoustic noise on actin in stereocilia of cochlear HCs. Fluorescent phalloidin, a highly specific F-actin stain, is used for staining the specimen. Intensity contributions from neighboring points are minimized without enhancing imaging noise. The analysis assumes that the relative intensity of a specific fluorescent label in stereocilia is proportional to the relative amount of F-actin present.

Stereocilia of guinea pigs are examined under three conditions: untreated control, sound-exposed without recovery, and sound-exposed with at least $10 \mathrm{~h}$ of recovery time. Using the phalloidin label, we have found several changes in the distribution of actin in stereocilia of sound-exposed groups compared to the control group. These changes may reflect either direct influence of noise exposure or an indirect response of HCs to the traumatic stimulus. The results demonstrate the usefulness of computational 3-D microscopy to analysis of stereocilia at the molecular level.

\section{Methods}

\section{Subjects}

Guinea pigs were divided into three groups. The first group was the untreated control $(N=5)$. The second group $(N=5)$ was subjected to acoustic stimulation (117 dB octave band noise at $1 \mathrm{kHz}$ for $4 \mathrm{~h}$ ) and sacrificed approximately $30 \mathrm{~min}$ following exposure. The third group $(N=5)$ was exposed to similar acoustic overstimulation but sacrificed between 10-15 h following noise exposure.

\section{Tissue preparation}

Guinea pigs in all groups were anesthetized and then sacrificed by decapitation. The temporal bones were removed and the cochleas were fixed for $2 \mathrm{~h}$ with $2 \%$ paraformaldehyde in $0.1 \mathrm{M}$ phosphate buffer at $\mathrm{pH}$ 7.35, permeabilized for $10 \mathrm{~min}$ in $0.3 \%$ Triton $\mathrm{X}-100$, and incubated for $30 \mathrm{~min}$ in rhodamine-conjugated phalloidin (Molecular Probes, OR) diluted 1:75 in PBS. The cochleas were then rinsed and dissected. The tectorial membrane was removed and the second and third turns of the organ of Corti, corresponding to the tonotopic region of the octave band noise centered at 1 $\mathrm{kHz}$, were mounted on slides in $60 \%$ glycerol in sodium carbonate buffer ( $\mathrm{pH} 8.5$ ) with p-phenylenediamine.

To count the number of stereocilia, they were oriented with their long axes perpendicular to the $X-Y$ plane. For this purpose, the cochlear tissue was placed in the gap between two coverslips placed as spacers on the microscope slide and a third coverslip placed on top. The stereocilia were not flattened under this arrangement. To measure the length and the fluorescence intensity along the stereocilia, spacers were not used and the stereocilia were flattened by the coverslip. This arrangement also minimized photobleaching of the phalloidin labeled specimen since fewer serial sections were sufficient to adequately image the stereocilia.

\section{Computational optical sectioning microscopy}

The specimens were examined in a Leitz Orthoplan microscope equipped with epifluorescence illumination. A Leitz Plan Apo objective lens $(100 \times ; 1.3$ N.A.; $170 / 0.17)$ was used for imaging the specimens. Images were acquired with a cooled charge-coupled device (CCD) camera (CC200 Photometrics, Inc.), shifting focus in $0.2 \mu \mathrm{m}$ steps using a piezoelectric translator (model P-286.40, Physik Instrumente). At an overall system magnification of $1,000 \times$, the equivalent size of the square pixel area of the CCD camera was $0.15 \times$ $0.15 \mu \mathrm{m}$.

A cooled CCD camera is an inherently linear device for light intensity (photoelectrons within a given pixel). Linearity was verified using a set of neutral density filters. The CCD dark current 'bad' pixels were controlled according to the procedure of Hiraoka et al. (1987). Astigmatism and other forms of optical distortion were assessed by the symmetry of the point spread function, and it was found that optical distortion was not a significant problem for the objective lens used in this study. The images were aligned using a computerized interactive registration procedure. Blur in the images was minimized using the computational optical sectioning microscopy procedure (COSMic) described in Avinash et al. (1992). This procedure requires knowledge of a function called the point spread function of the optical system. The appropriate point spread function was empirically determined by imaging rhodamine-coated microbeads of about $0.1 \mu \mathrm{m}$ diameter under a coverslip and mounting medium similar to that of the specimen. Serial images from individual stereocilia bundles were processed using the COSMic algorithm. Processed and unprocessed images were photographed from the computer monitor using a $35 \mu \mathrm{m}$ camera. Settings of the camera, the computer monitor, and the film processing were identical for both sets of images. 
Since the stereocilia diameters were smaller than 0.3 $\mu \mathrm{m}$ and less than two steps of focus, they occupied less than three pixels on the CCD sensor. An interactive program was used to determine the length of the stereocilia and the intensity along the length in 3-D. The average intensity of the three pixels across the diameter of stereocilia was used to assess this intensity. Thus, we have not attempted to accurately determine changes in the diameter of the stereocilia because they are at the resolution limit of optical microscopy. Intensity values for stereocilia are reported in a normalized fashion, i.e., in relation to the location as a percentage of their total length. This allowed direct comparison of stereocilia without regard to their absolute length.

\section{Results}

Three rows of OHCs and one row of inner hair cells and their stereocilia were clearly seen in our preparation. Only the observations on stereocilia of OHCs are reported here.

\section{Control cochleas (Group 1)}

In both Fig. 1 and Fig. 2, unprocessed and processed data are compared to demonstrate the ability of our technique to significantly remove blur. In the processed images, distinctive rows of stereocilia are clearly seen on a third turn $\mathrm{OHC}$ (Fig. 1) and on a second turn $\mathrm{OHC}$ (Fig. 2). The stereocilia never appeared bent under control conditions. Processed data visually indicate that the diameters of stereocilia in a given row are approximately equal but length was not rigorously measured in this study.

\section{Noise-exposed cochleas}

Extensive damage to the taller stereocilia was caused by a 4 hour noise exposure. With the octave band noise centered at $1 \mathrm{kHz}$, the maximum damage was found to occur in the upper second turn and lower third turn, as expected. The magnitude of visible damage as determined by stereocilia disarray progressively became less toward lower second turn and upper third turn. Appearance of first and fourth turn stereocilia, which were randomly inspected, was similar to stereocilia in
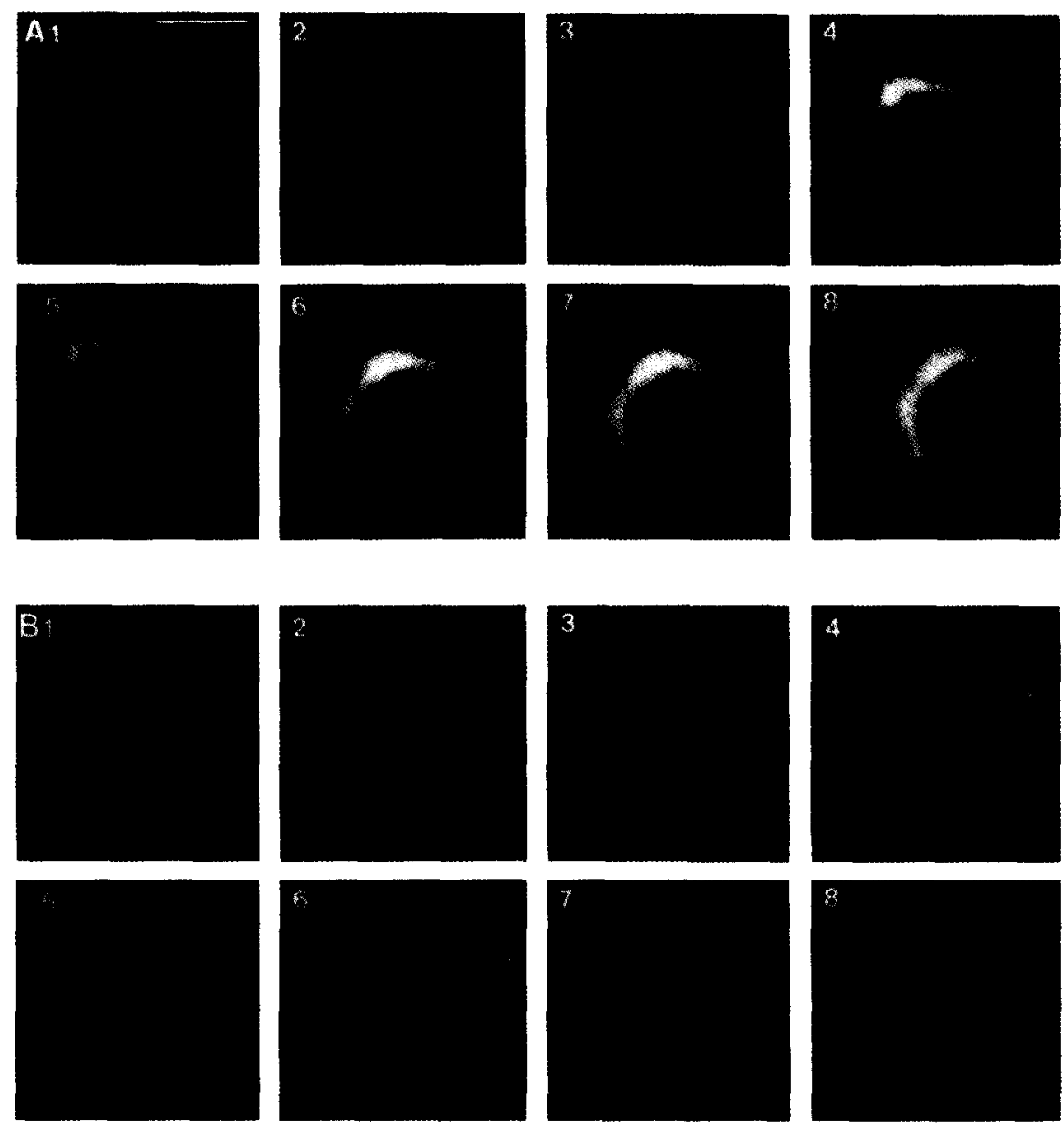

Fig. 1. Unprocessed (A) and processed (B) images of stereocilia from a third turn outer hair cell (group 1), oriented vertically. The separation between the displayed image sections is $0.8 \mu \mathrm{m}$. Bar, $2 \mu \mathrm{m}$. 

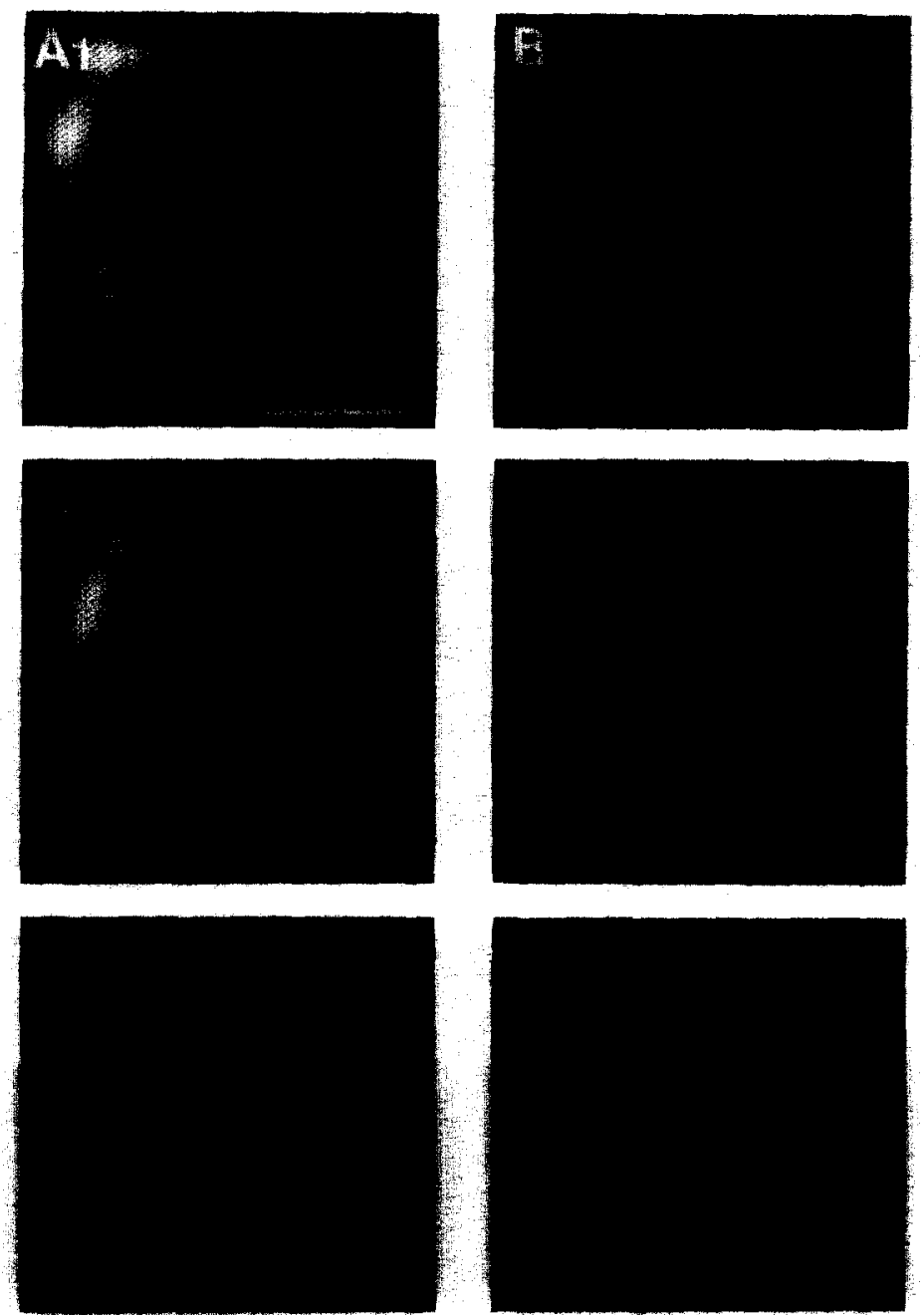

Fig. 2. Stereocilia of a second turn outer hair cell (group 1). (A): unprocessed data: (B): processed data. Image sections in series A and B are separated by $2 \mu \mathrm{m}$. Bar, $2 \mu \mathrm{m}$.

control cochleas at those locations. In the damaged region, stereocilia exhibited a variety of pathologies including a reduction in the number of tall stereocilia, variation in stereocilia length, variation in the distribution of label in the stereocilia bundle, and a variation in the intensity of label along the length of individual stereocilia. None of the pathologies appeared to be localized in a specific region of stereocilia and occasionally more than one type of damage was observed in a single cell.

\section{Group 2}

Surface views of these cochleas revealed a reduced number of tall stereocilia in second and third turn OHCs. Frequently, fluorescent images of stereocilia in the tallest row were about $50 \%$ shorter than adjacent stereocilia in the same row. In addition, elongated stereocilia were occasionally observed. In some normal-appearing cells without any measurable changes in length, some individual stereocilia appeared thinner than their neighbors in the same bundle. Noise damage in this group is summarized in Fig. 3.

\section{Group 3}

Although these cochleas were noise exposed with the same protocol as group 2, they displayed more damage in the stereocilia bundles. An unusually large number of broken stereocilia for this kind of tissue preparation was found scattered on the tissue. Many HCs in the second turn and the lower third turn were missing. Phalangeal scars were found in spaces previously filled by OHCs prior to their degeneration (data not shown). Some of the remaining OHCs had lost all or most of the taller stereocilia and also some smaller stereocilia; some stereocilia were significantly shortened. The surface of damaged OHCs was completely devoid of phalloidin label, suggesting that no stereocilia were present. The bundles of stereocilia often included unusually elongated stereocilia. Increase in 
length was accompanied by a reduction in the number of tall stereocilia or by thinncr-appcaring stcreocilia. Sometimes a single stereocilium was elongated, and occasionally as many as eight elongated stereocilia were found on a single $\mathrm{OHC}$. In a few images from group 3, stereocilia from one cell appeared interlocked with its neighbor's stereocilia. These results are summarized in Fig. 4.
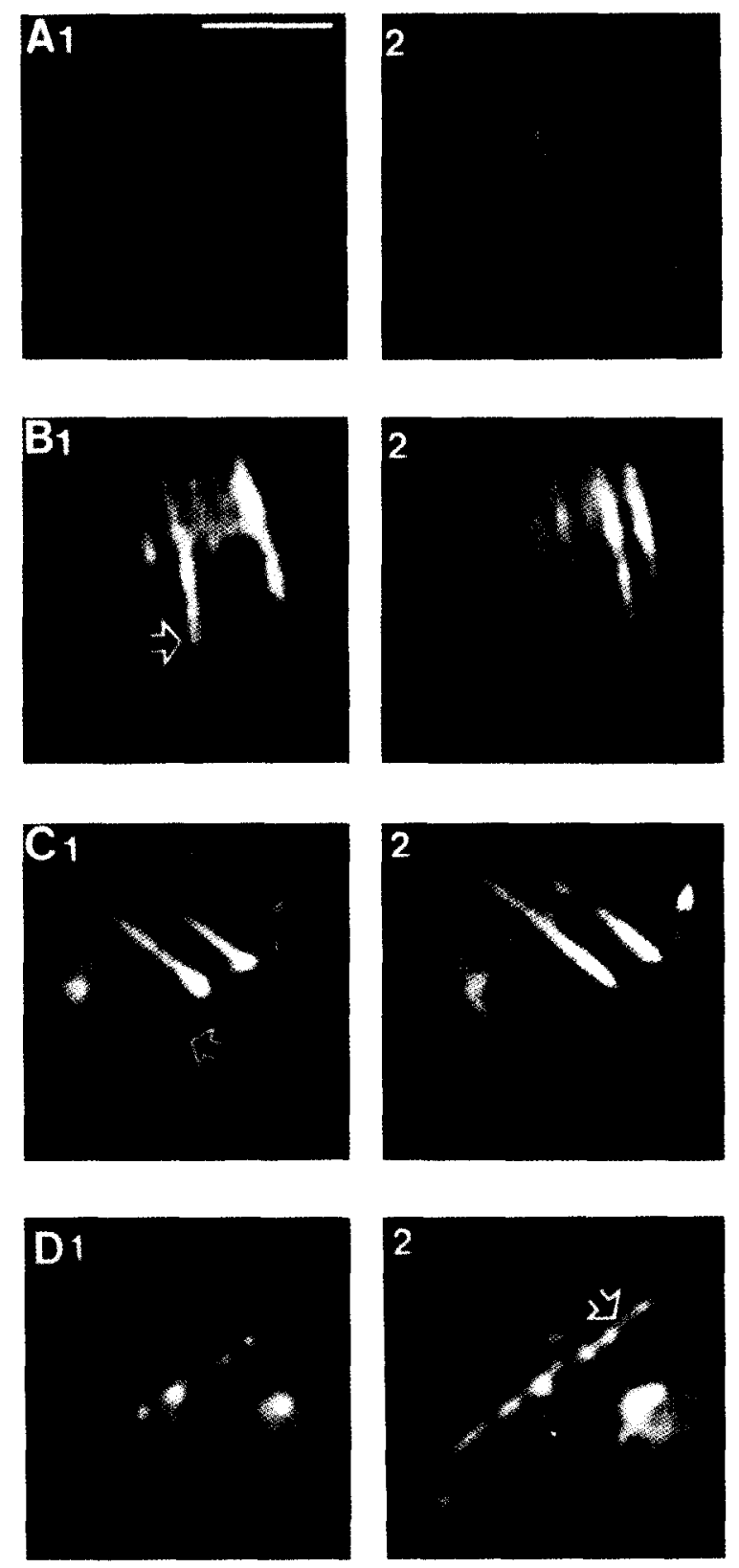

Fig. 3. Damage in stereocilia immediately after noise exposure (group 2). (A): reduced number of tall stereocilia; (B): shrunken tall stereocilia (arrow); (C): relatively thinner tall stereocilia (arrow); (D): increase in size of a tall stereocilium (arrow). The stereocilia is double in diameter and 50\% longer (complete length not shown). Image sections 1 and 2 in series A, B, C and D are separated by 0.6 $\mu \mathrm{m}$. Bar, $2 \mu \mathrm{m}$.
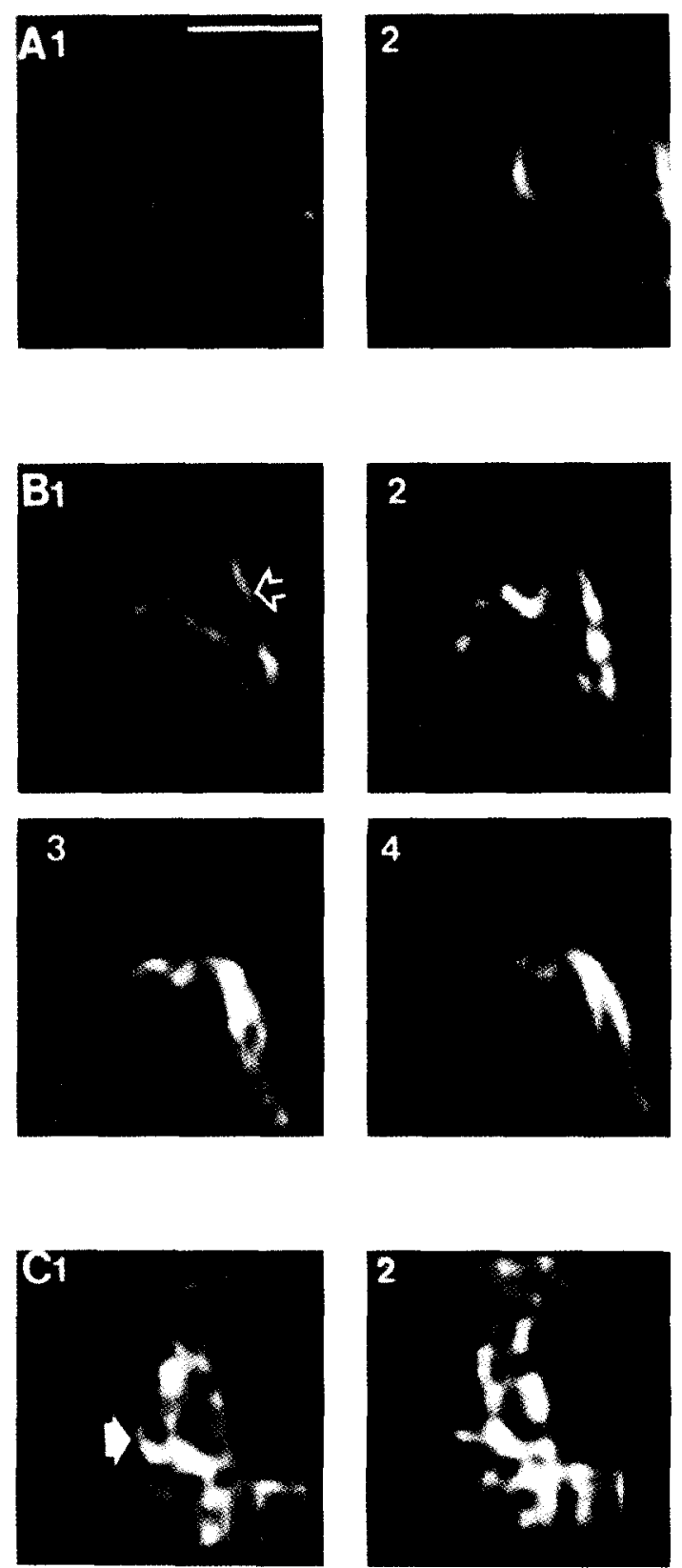

Fig. 4. Noise damage in stereocilia after noise exposure and 10-14 h of recovery (group 3). (A): Reduction in the stereocilia number. Note that bent stereocilia have reduced relative intensity. (B): Both elongation (arrow) and reduction in length of stereocilia in the same bundle. Near the bases of stereocilia in lower optical sections 3 and 4, there are no visible changes. (C): stereocilia from two different OHCs appear interlocked (solid arrow). Image sections 1 and 2 in series $\mathrm{A}$ and $\mathrm{C}$ and sections $1,2,3$ and 4 in series $\mathrm{B}$ are separated by $0.6 \mu \mathrm{m}$. Bar, $2 \mu \mathrm{m}$.

Many stereocilia in the damaged region appeared bent. A careful study of such stereocilia with 3-D analysis revealed that fluorescent intensity at the bends was relatively less than in other regions (Figs. 5A and $5 B)$. Thinner stereocilia exhibiting relatively lower intensity of label and variable label intensity along the length, were often noticed (Fig. 5C). 


\section{Label intensity along the length of stereocilia}

The intensity of actin labeling along the length of stereocilia was analyzed in the second and third turns. In unexposed cochleas, these stereocilia were labeled at approximately the same intensity, within the limits of image noise, for about $80 \%$ of their length from the tip. However, the intensity along the remaining $20 \%$ of length declined sharply (Fig. 6A). In spite of variations in length and thickness, stereocilia in group 2 showed a type of labeling pattern along their length similar to control stereocilia. In group 3, stereocilia which appeared bent were selected for analysis of actin distribution along their length. In Fig. 6B, the label intensities along four such stereocilia are shown. In these stereocilia, label intensity appeared diminished at the bends. There were more intensity variations along the length in stereocilia of the exposed groups than in the unexposed group. For clarity, in Fig. 6C representative
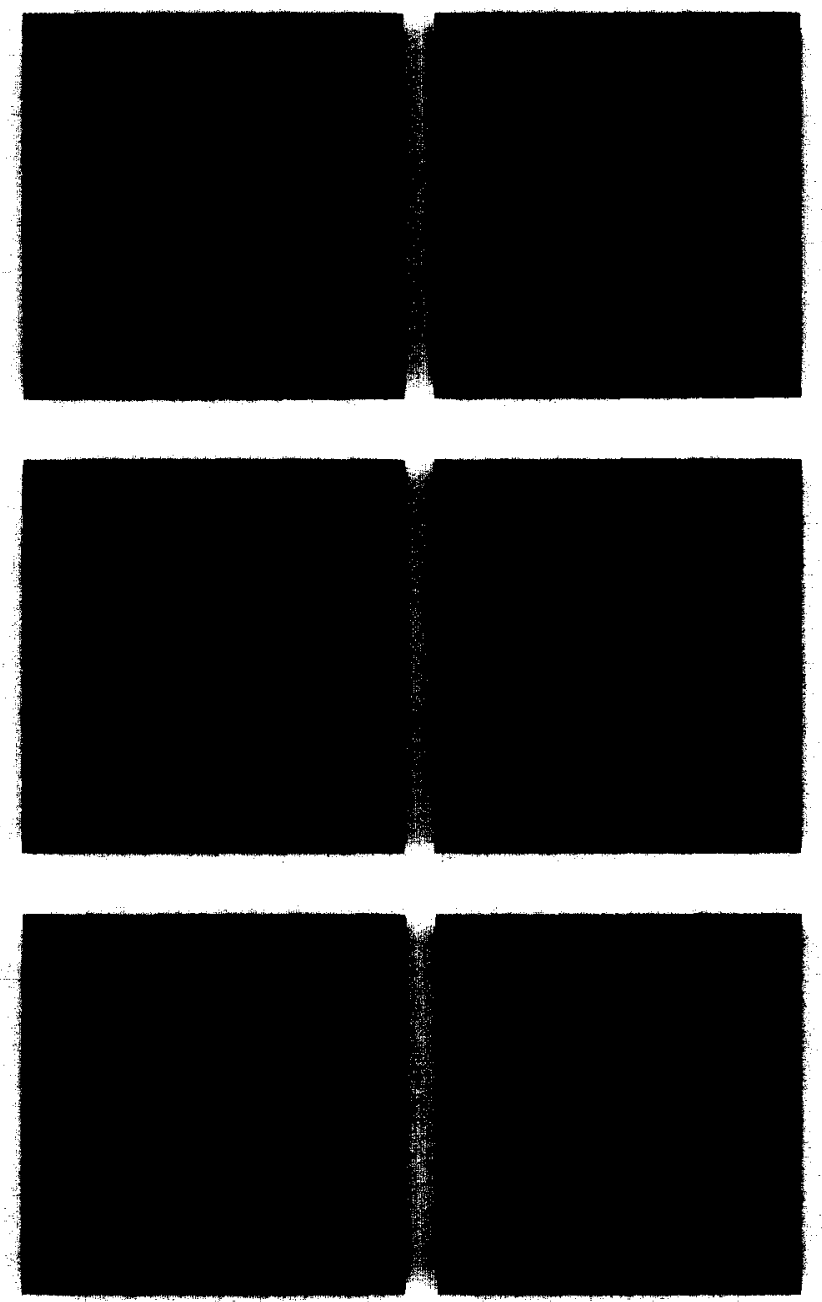

Fig. 5. Reduction in the fluorescent intensity in stereocilia on one $\mathrm{OHC}$ after noise damage and more than $10 \mathrm{~h}$ of recovery (group 3). (A) and (B): reduction of intensity in bent stereocilia (arrows). (C): variations in the diameter (arrow) and relatively thinner stereocilia which are less intensely labeled. Image sections 1 and 2 in series (A, B) and (C) are separated by $0.6 \mu \mathrm{m}$. Bar, $2 \mu \mathrm{m}$.
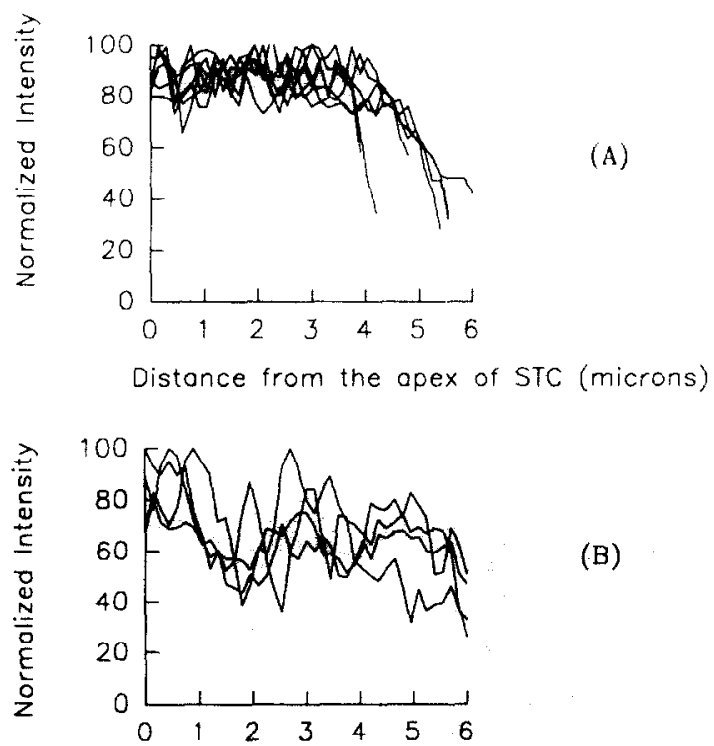

Distance from the opex of STC (microns)

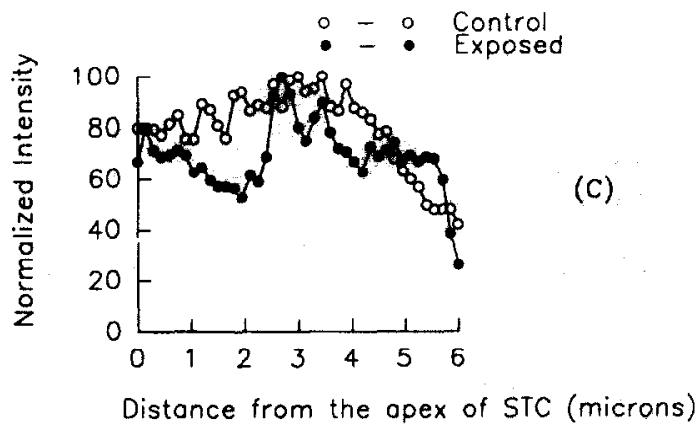

Fig. 6. Label intensity along the length of control (group 1) and noise-exposed (group 3) stereocilia, measured from the tip (apex) of stereocilia down to their base at the reticular lamina: (A) Fluorescence intensity along seven stereocilia from second and third turns of four control cochleas. (B) Fluorescence intensity along four bent stereocilia from three group 3 cochleas. (C) Comparison between a stereocilium of the control and another from an exposed cochlea. The decrease in the intensity at about $2 \mu \mathrm{m}$ from the tip of exposed stereocilia corresponds to the intensity at the bend.

stereocilia from control and noise-exposed group 3 are shown. The intensity along the length of the stereocilia indicated by an arrow in Fig. 5A is shown in Fig. 6C. Elongated stereocilia in group 3, however, did not show any variations in label intensity along their lengths (results not shown).

\section{Discussion}

A computational 3-D optical microscopy technique was used to quantitate fluorescent label in stereocilia by deblurring images without enhancing noise. Care was taken in the image processing to adhere to the model dictated by mathematical physics. It was assumed that the intensity of label in the images is an indicator of the amount of actin present. The present 
approach enhanced spatial and contrast resolution of label, thereby providing quantitative information at the molecular level. The analysis enabled us to accurately measure the changes in stereocilia length and intensity.

\section{Reduction in the number of stereocilia}

Reduction in the number of stereocilia was clearly seen in the noise-exposed cochleas. Noise exposure may weaken the otherwise stiff stereocilia by fracturing or depolymerizing actin at the base, so that stereocilia fall off during the exposure or during specimen preparation. The presence of an unusually large number of broken stcreocilia lying on the tissue supports fracturing of actin at the base of stereocilia. Previous studies have also reported a reduction in stereocilia number following noise exposure. It is not clear whether overstimulation causes reduction in stereocilia number directly, or whether these changes are secondary to pathological effects elsewhere in the HC.

\section{Variation in the length of stereocilia}

Reduction in length of some tall stereocilia may be related to actin depolymerization in the tip region of stereocilia. However, increase in length of stereocilia in some HCs may be due to the addition of actin. Stereocilia elongation can occur by the reorganization of pre-existing G-actin. Uniform intensity of label along the length of elongated stereocilia indicates a uniform actin distribution. Thinner stereocilia may be the result of a reduction of the number of actin filaments in the core, by depolymerization. The thinning of stereocilia and/or a reduction in the number of stereocilia accompanying elongated stereocilia seem to suggest that an existing pool of actin is reorganized, leading to the formation of elongated stereocilia. Alternatively, elongation of stereocilia could also result from a decrease in the normal rate of actin depolymerization.

It has been suggested that elongated stereocilia form in response to noise exposure, duc to the removal of capping molecules which normally prevent elongation (Raphael and Altschuler, 1992). The elongation may protect $\mathrm{OHCs}$ from noise trauma by uncoupling the tectorial membrane from the stereocilia of neighboring cells. A protective role for the giant stereocilia in waltzing guinea pig has been suggested by Canlon et al. (1989). Alternatively, the formation of the giant stereocilia may represent an attempt by a damaged cell to reorganize its actin cytoskeleton. Engström et al. (1983) documented small, thin kinocilia and giant stereocilia in rabbits with severe hearing loss and suggested that these cells were attempting to regenerate.

\section{Variation in actin label in stereocilia}

Variation in diameter of apparently straight stereocilia can be a result of reduction of F-actin due to depolymerization or difference in packing and cross linking the filaments. Tall stereocilia which were relatively thinner in the noise-damaged cochlea might have resulted from the depolymerization of some of the actin filaments that make up the actin core. In any given (noise-exposed) ear, different patterns of changes were present in the diameter of stereocilia, indicating that more than one mechanism may underlie these changes.

Variations in label intensity along the length of stereocilia

The uniformity of actin label in the upper $80 \%$ of the length of normal stereocilia demonstrates the ability of our method to detect uniform distribution of F-actin. The narrow actin bundle near the rootlet of the stereocilia appears as an area with reduced intensity in our analysis. This reflects the reduced number of actin filaments in the rootlet. Changes in the organization of actin may have occurred in the region of the rootlet, but our present equipment is not sensitive enough to apply the mathematical algorithm to this region. With the camera used for these experiments, at an optical magnification of $1000 \times$, each image pixel represents integrated fluorescence intensity from about $0.15 \times 0.15 \mu \mathrm{m}$ area on the specimen.

Reduction in fluorescent intensity at the bent portion of damaged stereocilia may correspond to the breaking of actin cross links at these places, whereas variations in the diameter of stereocilia along the length may be the manifestation of loss of F-actin due to depolymerization. Variations in intensity along the length of stereocilia were frequently observed in group 3 . We speculate that such changes are related to the active reorganization of $\mathrm{F}$-actin after acoustic overstimulation.

\section{Secondary events in noise trauma}

To better understand the mechanism of noise trauma, it is important to determine which changes are directly caused by the noise exposure and which changes are secondary. It is also important to distinguish between degenerative changes and changes that represent stress response allowing cells to survive and possibly to recover from the trauma. Elongated ('giant') stereocilia were previously observed on many surviving HCs several months after acoustic trauma (Engström et al., 1983). We, therefore, speculate that the presence of elongated stereocilia is related to stress response and does not necessarily lead to degeneration. In contrast, cells in group 2 with short or narrow stereocilia may correspond to cells in group 3 displaying signs of degeneration. It should be noted that these morphological changes include depolymerization of $\mathrm{F}$-actin and are likely to be regulated in a specific pattern of active cell death. This has been suggested previously, based on the observation that the apical belt of adherens 
junctions constricts in degenerating HCs (Raphael and Altschuler, 1992).

Since the damaged region was larger in group 3 as compared to group 2 animals, we conclude that depolymerization progresses even after the traumatizing acoustic stimulus is terminated. This also implies that some of the normal appearing stereocilia which were found adjacent to the lesion in group 2, may have belonged to damaged HCs. It is not clear whether changes were too subtle to be detected using our technique or changes in stereocilia were secondary and did not appear immediately after the noise exposure.

It is not known how the fate of HCs to recover or degenerate is determined. A more detailed study on the time course of deterioration by varying the duration of both noise exposure and recovery may help distinguish between primary and secondary events and determine the point of divergence between cellular survival and degeneration.

In conclusion, the computational 3-D microscopy technique was used to quantitatively study noise-induced damage in the stereocilia of guinea pig OHCs. Actin in auditory epithelia was labeled using rhodamine-conjugated phalloidin. Variations in length and intensity of label were observed in the noise-damaged stereocilia. These changes were neither limited to any particular region of stereocilia nor found to occur in any typical fashion. Elongation of stereocilia was always associated with reduction in the total number of stereocilia on a cell. This suggests that reorganization of the pre-existing pool of actin may possibly account for the elongation.

The thinning of stereocilia diameter or reduction in the number of stereocilia in a bundle whenever an elongated stereocilia appeared suggests that an existing pool of actin is reorganized after noise exposure. Damage was more pronounced $10-15 \mathrm{~h}$ following the noise exposure compared to immediately after the exposure, suggesting that at least part of the damage in stereocilia was a secondary response. Some of these degenerative changes may be actively regulated. The results indicate that the study of normal and noise-damaged stereocilia is possible with optical microscopy, after reduction of distortions in 3-D images using our technique.

\section{Acknowledgments}

The research is supported by NIH grants DC 000105 , DC 00078 and a Deafness Research Foundation grant (YR). We thank Dr. Yu Wang and Mr. Michael K. Lee for technical assistance.

\section{References}

Avinash, G., Nuttall, A.L., O'Donnell, M. and Wakefield, G.H. (1993) Quantitative three-dimensional optical microscopy using convex projection theory. IEEE Trans. Med. Imaging (in press).

Canlon, B., Flock, $\AA$. and Borg, E. (1989) Can giant stereocilia protect the organ of Corti from noise damage? Ahstr. Assoc. Res. Otolaryngol. St. Petersburg Beach, FL.

Engström, B., Flock, $\AA$. and Borg, E. (1983) Ultrastructural studies of stereocilia in noise-exposed rabbits. Hear. Res. 12, 251-264.

Flock, $\AA$. and Cheung, H.C. (1977) Actin filaments in sensory hairs of inner ear receptor cells. J. Cell Biol. 75, 339-343.

Hiraoka, Y., Sedat, J.W. and Agard, D.A. (1987) The use of a charge-coupled device for quantitative optical microscopy of biological structures. Science 238, 36-41.

Korn, E.D., Carlier, M-F. and Pantaloni, D. (1987) Actin polymerization and ATP hydrolysis. Science 238, 638-644.

Liberman, M.C. (1987) Chronic ultrastructural changes in acoustic trauma: Serial section reconstruction of stereocilia and cuticular plates. Hear. Res. 26, 65-88.

Liberman, M.C. and Dodds, L.W. (1987) Acute ultrastructural changes in acoustic trauma: Serial section reconstruction of stereocilia and cuticular plates. Hear. Res. 26, 45-64.

Raphael, Y. and Altschuler, R.A. (1991) Reorganization of cytoskeletal and junctional proteins during cochlear hair cell degeneration. Cell Motil. Cytoskeleton 18, 215-227.

Raphael, Y. and Altschuler, R.A. (1992) Early microfilament regeneration in injured auditory epithelia. Exp. Neurol. 115, 32-36.

Saunders, J.C., Dear, S.P. and Schneider, M.E. (1985) The anatomical consequences of acoustic injury: A review and tutorial. J. Acoust. Soc. Am. 78, 833-860.

Saunders, J.C., Cohen, Y.E. and Szymko, Y.M. (1991) The structural and functional consequences of acoustic injury in the cochlea and peripheral auditory system: A five year update. J. Acoust. Soc. Am. 90, 136-146.

Tilney, L.G., Saunders, J.C., Egelman, E. and DeRosier, D.J. (1982) Changes in the organization of actin filaments in the stereocilia of noise-damaged lizard cochleae. Hear. Res. 7, 181-97. 